\section{Higher enhanced computed tomography attenuation value of the aorta is a predictor of massive transfusion in blunt trauma patients}

\author{
Tetsuya Yumoto, Hiromi Ihoriya, Ryo Tanabe, Hiromichi Naito, \\ Atsunori Nakao
}

Department of Emergency, Critical Care, and Disaster Medicine, Okayama University Graduate School of Medicine, Dentistry and Pharmaceutical Sciences, Okayama, Japan

Objective Several scoring systems have been developed to identify patients who require massive transfusion (MT) after major trauma to improve survival. The primary goal of this study was to investigate the usefulness of enhanced computed tomography attenuation values (CTAVs) of major vessels to determine the need for MT in patients with major blunt trauma.

Methods This single-center retrospective cohort study evaluated patients aged 16 years or older who underwent contrast-enhanced computed tomography scan of the torso after major blunt trauma. The CTAVs of six major vessel points in both the arterial and portal venous phases at initial computed tomography examination were assessed and compared between the MT and the no MT group. The capability of enhanced CTAVs to predict the necessity for MT was estimated based on the area under the receiver operating characteristic curve.

Results Of the 254 eligible patients, 36 (14\%) were in the MT group. Patients in the MT group had significantly higher CTAVs at all sites except the inferior vena cava in both the arterial and portal venous phases than that in the no MT group. The descending aorta in the arterial phase had the highest accuracy for predicting MT, with an AUROC of 0.901 (95\% confidence interval, 0.855 to $0.947 ; P<0.001)$.

Conclusion Initial elevation of enhanced CTAV of the aorta is a predictor for the need for MT. A higher CTAV of the aorta should alert the trauma surgeon or emergency physician to activate their MT protocol.

Keywords Computed tomography attenuated value; Massive transfusion; Wounds and injuries
eISSN: 2383-4625

Received: 28 December 2018

Revised: 12 February 2018

Accepted: 25 February 2018

Correspondence to: Tetsuya Yumoto Department of Emergency, Critical Care, and Disaster Medicine, Okayama University Graduate School of Medicine, Dentistry and Pharmaceutical Sciences, 2-5-1 Shikata-cho, Kita-ku, Okayama 700-8558, Japan

E-mail: tyumoto@cc.okayama-u.ac.jp ORCID

https://orcid.org/0000-0003-1766-8026

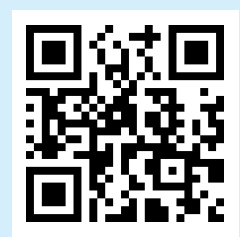

How to cite this article:

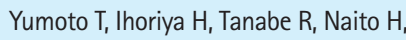
Nakao A. Higher enhanced computed tomography attenuation value of the aorta is a predictor of massive transfusion in blunt trauma patients. Clin Exp Emerg Med 2019;6(4):330-339.
This is an Open Access article distributed under the terms of the Creative Commons Attribution Non-Commercial License (http:// creativecommons.org/licenses/by-nc/4.0/). 


Capsule
Wummat is already known
Several scoring systems have been developed to predict the need for massive transfusion (MT) in severely injured trau-
ma patients. Although whole-body computed tomography scanning in trauma patients is common, the significance of
enhanced computed tomography attenuation values (CTAVS) of major vessels to predict the need for MT has not been
elucidated.
What is new in the current study
In this study, we found a strong association between initial enhanced CTAV of the aorta and the necessity for MT in ma-
jor blunt trauma patients. A simple measurement of CTAV of the ascending or descending aorta in the arterial phase,
which had the highest interobserver agreement and accuracy, could predict the MT requirement.

\section{INTRODUCTION}

Major hemorrhage remains a leading cause of early death after severe trauma. ${ }^{1}$ In addition to immediate identification of bleeding sources and the appropriate hemostatic intervention, early transfusion can prevent the development of acute traumatic coagulopathy and improve survival. ${ }^{2-5}$ Delayed recognition and control of active hemorrhage is among the major causes of preventable death from trauma. ${ }^{6}$

Several scoring systems have been developed to predict the need for massive transfusion (MT) in severely injured trauma patients. These systems incorporate several clinical parameters including age, vital signs, laboratory data, and ultrasound or pelvic X-ray findings. ${ }^{7-12}$ As whole-body computed tomography (CT) scanning in severely injured trauma patients has been shown to yield beneficial outcomes and is commonly performed in current trauma practice, specific CT findings may be helpful in determining the need for MT. The inferior vena cava (IVC) diameter on the initial CT image has been reported to be a potential predictor of mortality or the necessity for MT. ${ }^{2,13-15}$

Although decreased pancreatic perfusion in the early phase and delayed pancreatic enhancement in the late phase have been shown to be associated with hypovolemic shock, the significance of enhanced computed tomography attenuation values (CTAVs) of major vessels has not been elucidated. ${ }^{16}$ Therefore, this study aimed to investigate the usefulness of enhanced CTAV of major vessels as a predictor of MT in patients with major blunt trauma. We hypothesized that the enhanced CTAV of major vessels such as the aorta would be higher in patients requiring MT due to lower cardiac output.

\section{METHODS}

\section{Study design and participants}

This single-center retrospective study reviewed the trauma registry and medical records of consecutive trauma patients admitted to our tertiary hospital between April 2014 and March 2018. Patients younger than 16 years old and those with cardiac arrest on arrival, penetrating mechanism of injury, Injury Severity Score of less than 9, and those who had not undergone contrast-enhanced CT scans of the torso were excluded. Patients who had been administered contrast material intravenously through the lower extremity were also excluded. This study was approved by the ethics committee of Okayama University Hospital (1807-015). The requirement for written informed consent was waived due to the retrospective nature of the study.

\section{Patient management}

In our hospital, whole-body CT scanning, including native CT examination of the head, neck, and torso (i.e., chest, abdomen, and pelvis), is performed during the initial evaluation of blunt torso trauma patients, regardless of hemodynamic status. This is followed by enhanced CT scanning of the torso in hemodynamically unstable patients and those with high-energy trauma. After bleeding sources are identified and the hemorrhage is successfully controlled via surgical and/or catheter intervention, the necessity for MT is determined based on clinical judgment. Patients who are identified to need MT received transfusions of red blood cells (RBCs), fresh frozen plasma, and platelet concentrates at a 1:1:1 ratio as soon as possible.

\section{CT examination protocol}

CT imaging was performed using an Aquilion 64-row multi-slice 
CT (Toshiba Medical Systems, Okayama, Japan) with the following imaging parameters: tube voltage, $120 \mathrm{kV}$; tube current, $300 \mathrm{~mA}$; slice thickness, $5 \mathrm{~mm}$; and gantry rotation time, 0.75 seconds. After obtaining native scans of the head, neck, and torso (i.e., chest, abdomen, pelvis), contrast-enhanced CT of the torso combining the arterial and portal venous phases was obtained. A total of either $100,125,135$, or $150 \mathrm{~mL}$ contrast material and iodine concentrations of either 300, 350, or $370 \mathrm{mg}$ iodine/mL (ideally, 500 to $600 \mathrm{mg}$ iodine $/ \mathrm{kg}$ ) were administered within 30 seconds through peripheral venous access in the upper extremity based on the estimated body weight. Patients underwent scans of 5-mm-thick sections of the torso during the arterial and portal venous phases, which were conducted 30 and 80 seconds after the start of the injection of contrast material, respectively.

\section{CT measured contrast attenuation values of the major vessels}

The mean Hounsfield unit (HU) density was quantified by using an elliptical region-of-interest tool of a commercially available viewer software package (Synapse ver. 4.1, Fujifilm Medical, Tokyo, Japan). ${ }^{16-18}$ Given the measurability and application for clinical prac- tice, we selected six major vessel points in both the arterial and portal venous phases to determine predictability and reliability, namely, 1) the main pulmonary artery at the level of its bifurcation; 2,3 ) the ascending aorta and descending aorta at the level of main pulmonary artery bifurcation; 4) the abdominal aorta at the left renal artery branching level; 5) the common femoral artery on the right; and 6) the IVC $25 \mathrm{~mm}$ (5 slices) above the right renal vein (Fig. 1). Cross-sectional largest possible areas of each vessel were measured, avoiding the edges of the vessels to prevent partial volume effect caused by calcified plaques. ${ }^{16-18}$ The left common femoral artery was evaluated to confirm correct placement of the catheter. The CTAV of IVC or the abdominal aorta was traced, avoiding the area where the catheter had been placed.

CTAVs were measured twice by two emergency physicians (a senior resident and an attending physician) who were unaware of the patient's outcomes. The means of the two measurements were then analyzed.

\section{Data collection}

Demographic information and clinical data, including mechanism of injury, vital signs, base excess, lactate level, coagulation mark-
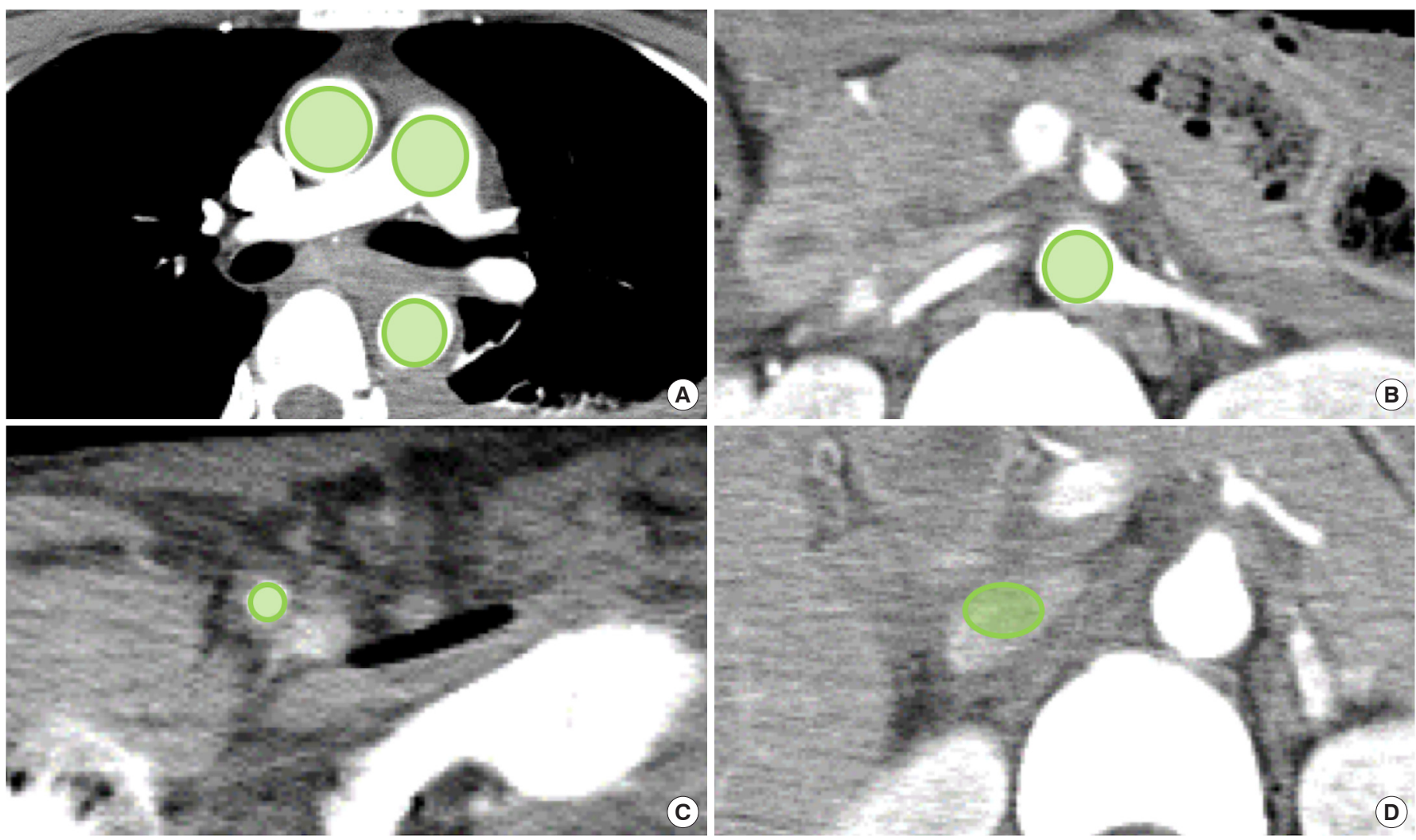

Fig. 1. Six points of measurement of enhanced computed tomography attenuation values: 1) main pulmonary artery at the level of its bifurcation, 2, 3) ascending aorta and descending aorta at the same level (A), 4) abdominal aorta at the left renal artery branching level (B), 5) common femoral artery on the right (C), and 6) inferior vena cava $25 \mathrm{~mm}$ above the right renal vein (D). 
ers on emergency department arrival, Injury Severity Score, associated injuries, blood products received, treatment, patient outcomes, and enhanced CTAV of major vessels, were collected. As the degree of contrast enhancement is affected by body weight and total iodine used, an additional analysis was conducted using CTAV adjusted by iodine dose per body weight. ${ }^{19}$ MT was defined as a transfusion of 8 or more units of RBCs within the first 6 hours after the patient's arrival at the hospital because the traditional MT concept (10 units or more of RBCs within 24 hours) has been shown to be imprecise due to survivorship bias. ${ }^{20-22}$

\section{Statistical analysis}

Continuous variables were described as median and interquartile range and categorical variables as frequencies or percentages. Univariate analyses were performed using Fisher exact probability test and Mann-Whitney U-test as appropriate. Intraclass correlation analysis was used to determine the interobserver agreement of the enhanced CTAV measurements. Spearman correlation coefficients were computed to compare the relationship between enhanced CTAV and physiological parameters. The capability of enhanced CTAV to predict the necessity for MT was estimated based on the area under the receiver operating characteristic curve (AUROC). The optimal cut-off value was explored using the Youden index. A two-tailed P-value of $<0.05$ was considered statistically significant. All analyses were conducted using IBM SPSS Statistics ver. 25.0 (IBM Corp., Armonk, NY, USA).

\section{RESULTS}

\section{Study population and characteristics}

In total, 679 trauma patients were admitted to our tertiary hospital during the study period; of these, we excluded 425 patients and thus 254 were included in the analysis. The MT group and the no MT group comprised 36 and 218 patients, respectively. Three patients without contrast-enhanced CT scans of the torso, one patient with aortic injury, and two patients with catastrophic brain injury died. However, no patients died due to exsanguination without receiving MT.

The baseline patient characteristics are summarized in Table 1. Patients who required MT presented with significantly higher rates of older age, more severe injury with respect to physiological and anatomical parameters, and higher rates of hemostatic interventions than those without MT. Although no significant differences in CTAV of the IVC in the arterial phase were observed between the two groups, patients in the MT group had significantly higher CTAV at all other sites in both the arterial and portal venous phases than the no MT group.

\section{Interobserver reliability of CTAV measurements}

Interobserver reliability was found to be excellent, with intraclass correlation coefficients of 0.995 and $0.984 ; 0.994$ and $0.995 ; 0.996$ and $0.983 ; 0.993$ and $0.968 ; 0.955$ and $0.934 ;$ and 0.940 and 0.909 for the CTAVs of the pulmonary artery; ascending aorta; descending aorta; abdominal aorta; femoral artery; and IVC in the arterial and portal venous phases, respectively.

\section{Correlation between enhanced CTAVs of major vessels and clinical parameters on arrival or requirement of RBCs within 24 hours}

Table 2 shows the correlation between CTAV of major vessels and representative physiological parameters including heart rate, systolic blood pressure (SBP), lactate level on arrival, or requirement of RBCs within 24 hours after arrival. A weak negative correlation was found between SBP and CTAV of all sites in both phases except for IVC in the arterial phase. Meanwhile, a weak positive correlation was observed between lactate level and CTAV of the aorta, except for the ascending aorta in the portal venous phase. RBCs required within 24 hours after arrival were moderately correlated with the CTAV of major vessels in both phases except for the IVC. Fig. 2 shows the correlation between CTAV of the ascending aorta in the arterial phase and the four parameters. CTAV of the ascending aorta in the arterial phase showed the strongest correlation with $\mathrm{RBC}$ r required within 24 hours $(r=0.568, \mathrm{P}<0.001)$.

\section{Predictive values of enhanced CTAVs for MT}

The accuracy of enhanced CTAV of major vessels and other variables as predictors for MT was assessed (Table 3). Enhanced CTAV of major vessels except for the IVC showed moderate accuracy, with an AUROC greater than 0.7. The descending aorta in the arterial phase had the highest accuracy; the AUROC was 0.901 (95\% confidence interval $[\mathrm{Cl}], 0.855$ to $0.947 ; \mathrm{P}<0.001$ ) (Fig. 3A). The cut-off that maximized the Youden index was $304 \mathrm{HU}$, with a sensitivity, specificity, positive likelihood ratio, and negative likelihood ratio of 0.889 (95\% Cl, 0.759 to 0.955$), 0.757$ (95\% Cl, 0.735 to 0.768$), 3.656(95 \% \mathrm{Cl}, 2.868$ to 4.114$)$, and $0.147(95 \% \mathrm{Cl}$, 0.058 to 0.328$)$, respectively.

Similarly, the CTAV of major vessels adjusted by the iodine dose per body weight was also confirmed to be a moderate predictor for MT. The ascending aorta in the arterial phase had the highest accuracy; the AUROC was $0.863(95 \% \mathrm{Cl}, 0.799$ to $0.927 ; \mathrm{P}<0.001)$ (Fig. 3B). The cut-off that maximized the Youden index was 0.57 $\mathrm{HU}^{*} \mathrm{~kg} / \mathrm{mg}$, with a sensitivity, specificity, positive likelihood ratio, and negative likelihood ratio of $0.750(95 \% \mathrm{Cl}, 0.603$ to 0.859$)$, 0.697 ( $95 \% \mathrm{Cl}, 0.673$ to 0.715$), 2.477(95 \% \mathrm{Cl}, 1.843$ to 3.015$)$, and $0.359(95 \% \mathrm{Cl}, 0.198$ to 0.590$)$, respectively. 
Table 1. Baseline patient characteristics

\begin{tabular}{|c|c|c|c|c|}
\hline Characteristics & All patients $(n=254)$ & MT group $(n=36)$ & No MT group $(n=218)$ & P-value \\
\hline Age (yr) & $50(32-69)$ & $67(44-78)$ & $48(29-67)$ & 0.006 \\
\hline Male, sex & $179(70.0)$ & $25(69.4)$ & $154(70.6)$ & 0.85 \\
\hline Mechanism of injury & & & & 0.51 \\
\hline Motor vehicle accident & $186(73.2)$ & $27(75.0)$ & $159(72.9)$ & \\
\hline Fall & $55(21.7)$ & 6 (16.7) & 49 (22.5) & \\
\hline Others & $13(5.1)$ & $3(8.3)$ & $10(4.6)$ & \\
\hline Body weight (kg) & $60.9(54.0-70.1)$ & $58.8(52.0-72.9)$ & $60.9(54.8-69.9)$ & 0.44 \\
\hline lodine concentration (mg/mL) & $300(300-300)$ & $300(300-300)$ & $300(300-300)$ & 0.34 \\
\hline Contrast material dose $(\mathrm{mL})$ & $100(100-100)$ & $100(100-100)$ & $100(100-100)$ & 0.98 \\
\hline Total iodine used $\left({ }^{*} 10^{2} \mathrm{mg}\right)$ & $300(300-370)$ & $300(300-370)$ & $300(300-370)$ & 0.62 \\
\hline lodine dose per body weight $(\mathrm{mg} / \mathrm{kg})$ & $535(481-588)$ & $552(491-603)$ & $531(480-586)$ & 0.31 \\
\hline PA_A (HU) & $275(229-350)$ & $399(292-507)$ & $266(224-315)$ & $<0.001$ \\
\hline AsA_A (HU) & $275(241-347)$ & $414(346-466)$ & $265(236-316)$ & $<0.001$ \\
\hline DeA_A (HU) & $271(236-338)$ & $410(338-475)$ & $260(232-302)$ & $<0.001$ \\
\hline$A b A \_A(H U)$ & $279(238-341)$ & $383(330-479)$ & $266(232-319)$ & $<0.001$ \\
\hline$F A \_A(H U)$ & $261(221-313)$ & $337(269-417)$ & $252(213-302)$ & $<0.001$ \\
\hline IVC_A (HU) & 105 (82-133) & $103(62-158)$ & $105(84-131)$ & 0.79 \\
\hline PA_V (HU) & $152(133-175)$ & $182(165-204)$ & $147(130-167)$ & $<0.001$ \\
\hline AsA_V (HU) & $157(137-178)$ & $183(173-227)$ & $153(133-172)$ & $<0.001$ \\
\hline DeA_V (HU) & $157(140-178)$ & $187(168-224)$ & $153(135-174)$ & $<0.001$ \\
\hline AbA_V (HU) & $153(135-171)$ & $184(161-208)$ & $149(132-164)$ & $<0.001$ \\
\hline FA_V (HU) & $138(122-159)$ & $166(148-196)$ & $135(120-154)$ & $<0.001$ \\
\hline IVC_V (HU) & $128(113-144)$ & $148(127-179)$ & $125(112-140)$ & $<0.001$ \\
\hline Heart rate (beats/min) & 87 (75-104) & $109(89-125)$ & $84(74-100)$ & $<0.001$ \\
\hline Systolic blood pressure $(\mathrm{mmHg})$ & $126(107-150)$ & $88(64-110)$ & $131(113-154)$ & $<0.001$ \\
\hline Respiratory rate (cpm) & $21(19-25)$ & $22(19-28)$ & $20(19-24)$ & 0.21 \\
\hline Glasgow Coma Scale score & $14(11-15)$ & $12(5-14)$ & $14(11-15)$ & $<0.001$ \\
\hline Base excess (mmol/L) & $-1.7(-3.9-0.1)$ & $-6.6(-9.6--1.9)$ & $-1.2(-3.2-0.2)$ & $<0.001$ \\
\hline Lactate (mmol/L) & $2.4(1.7-3.4)$ & $3.7(2.9-5.6)$ & $2.2(1.5-3.1)$ & $<0.001$ \\
\hline Hemoglobin ( $\mathrm{g} / \mathrm{dL}$ ) & $13.5(11.6-14.5)$ & $11.6(9.7-13.5)$ & $13.8(12.0-14.8)$ & $<0.001$ \\
\hline Platelets $\left({ }^{*} 10^{4} / \mu \mathrm{L}\right)$ & $21.9(17.6-25.8)$ & $20.5(15.4-25.2)$ & $22.5(17.6-25.9)$ & 0.18 \\
\hline PT-INR & $1.01(0.96-1.07)$ & $1.08(1.02-1.22)$ & $1.00(0.96-1.05)$ & $<0.001$ \\
\hline Fibrinogen (mg/dL) & $233(190-275)$ & $163(125-221)$ & 240 (204-282) & $<0.001$ \\
\hline D-dimer $(\mu \mathrm{g} / \mathrm{mL})$ & $24.9(8.8-60.8)$ & $84.6(42.4-108.0)$ & $21.6(7.1-48.3)$ & $<0.001$ \\
\hline Positive FAST & $23(9.1)$ & $15(41.7)$ & $8(3.7)$ & $<0.001$ \\
\hline Pelvic fractures & $72(28.3)$ & $20(55.6)$ & $52(23.9)$ & $<0.001$ \\
\hline Head AIS $\geq 4$ & $73(28.7)$ & $14(38.9)$ & $59(27.1)$ & 0.17 \\
\hline Thoracotomy & $9(3.6)$ & $6(16.7)$ & $3(1.4)$ & $<0.001$ \\
\hline Laparotomy & $12(4.7)$ & $6(16.7)$ & $6(2.8)$ & 0.003 \\
\hline TAE & $26(10.2)$ & $17(47.2)$ & $9(4.1)$ & $<0.001$ \\
\hline RBC (units/24 hr) & $0(0,6)$ & $18(13,27)$ & $0(0,2)$ & $<0.001$ \\
\hline FFP (units/24 hr) & $0(0,6)$ & $20(14,33)$ & $0(0,4)$ & $<0.001$ \\
\hline PC (units/24 hr) & $0(0,0)$ & $20(10,30)$ & $0(0,0)$ & $<0.001$ \\
\hline ISS & $22(14-29)$ & $37(28-44)$ & $18(13-29)$ & $<0.001$ \\
\hline Hospital mortality & $15(5.9)$ & $9(25.0)$ & $6(2.8)$ & $<0.001$ \\
\hline
\end{tabular}

Values are presented as median and interquartile range or number (\%).

MT, massive transfusion; PA_A, pulmonary artery in the arterial phase; AsA_A, ascending aorta in the arterial phase; DeA_A, descending aorta in the arterial phase; $A b A \_A$, abdominal aorta in the arterial phase; FA_A, femoral artery in the arterial phase; IVC_A, inferior vena cava in the arterial phase; PA_V, pulmonary artery in the portal venous phase; AsA_V, ascending aorta in the portal venous phase; DeA_V, descending aorta in the portal venous phase; AbA_V, abdominal aorta in the portal venous phase; FA_V, femoral artery in the portal venous phase; IVC_V, inferior vena cava in the portal venous phase; PT-INR, prothrombin time-international normalized ratio; FAST, focused assessment with sonography for trauma; AIS, Abbreviated Injury Scale; TAE, transcatheter arterial embolization; RBC, red blood cell; FFP, fresh frozen plasma; PC, platelet concentrates; ISS, Injury Severity Score. 


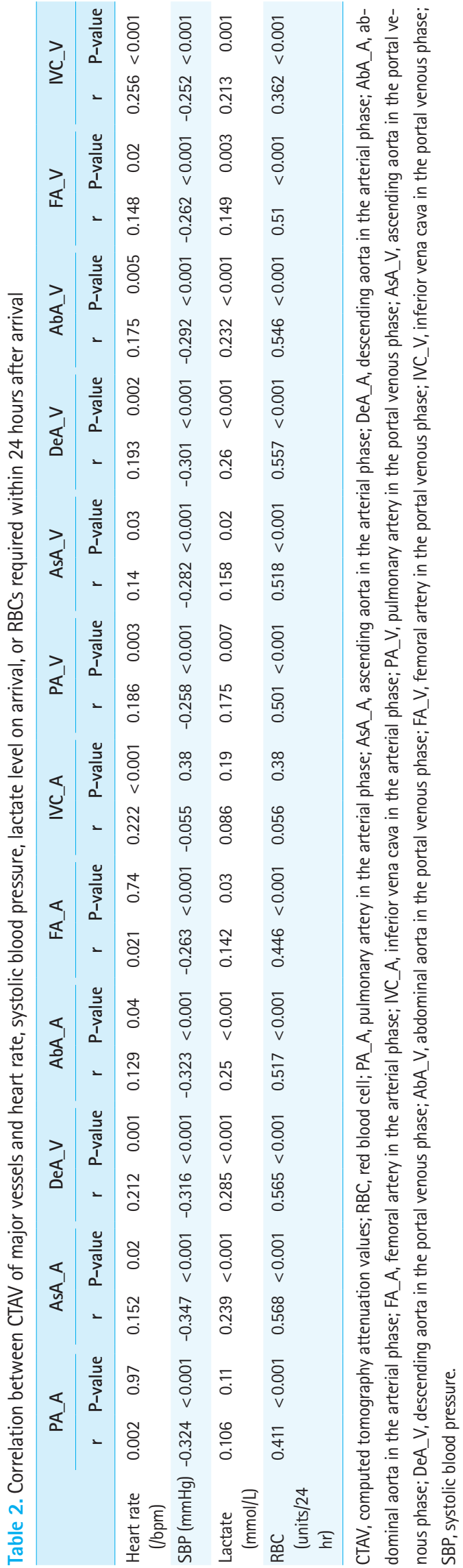

Table 3. AUROC values of absolute CTAV ${ }^{\text {a) }}$ of major vessels and other variables as predictors for massive transfusion

\begin{tabular}{|c|c|c|c|}
\hline Variable & AUROC $(95 \% \mathrm{Cl})$ & P-value & $\begin{array}{c}\text { Cut-off } \\
\text { value }\end{array}$ \\
\hline Heart rate (bpm) & $0.736(0.639-0.833)$ & $<0.001$ & 114 \\
\hline Systolic blood pressure (mmHg) & $0.834(0.758-0.909)$ & $<0.001$ & 113 \\
\hline Base excess (mmol/L) & $0.752(0.651-0.854)$ & $<0.001$ & -3.2 \\
\hline Lactate $(\mathrm{mmol} / \mathrm{L})$ & $0.768(0.688-0.849)$ & $<0.001$ & 2.9 \\
\hline Fibrinogen (mg/dL) & $0.767(0.665-0.869)$ & $<0.001$ & 192 \\
\hline D-dimer $(\mu \mathrm{g} / \mathrm{mL})$ & $0.797(0.716-0.878)$ & $<0.001$ & 40.0 \\
\hline PA_A (HU) & $0.786(0.697-0.876)$ & $<0.001$ & 322 \\
\hline AsA_A (HU) & $0.899(0.851-0.947)$ & $<0.001$ & 327 \\
\hline DeA_A (HU) & $0.901(0.855-0.947)$ & $<0.001$ & 304 \\
\hline AbA_A (HU) & $0.865(0.810-0.920)$ & $<0.001$ & 288 \\
\hline FA_A (HU) & $0.775(0.688-0.862)$ & $<0.001$ & 303 \\
\hline IVC_A (HU) & $0.486(0.363-0.610)$ & 0.85 & 73 \\
\hline PA_V (HU) & $0.830(0.761-0.899)$ & $<0.001$ & 157 \\
\hline AsA_V (HU) & $0.814(0.741-0.887)$ & $<0.001$ & 172 \\
\hline DeA_V (HU) & $0.827(0.767-0.887)$ & $<0.001$ & 162 \\
\hline AbA_V (HU) & $0.838(0.775-0.902)$ & $<0.001$ & 172 \\
\hline FA_V $(H U)$ & $0.805(0.735-0.874)$ & $<0.001$ & 155 \\
\hline IVC_V (HU) & $0.734(0.639-0.830)$ & $<0.001$ & 125 \\
\hline \multicolumn{4}{|l|}{$\begin{array}{l}\text { Adjusted values according to } \\
\text { iodine dose per body weight }\end{array}$} \\
\hline PA_A (HU*kg/mg) & $0.761(0.663-0.858)$ & $<0.001$ & 0.60 \\
\hline AsA_A (HU*kg/mg) & $0.863(0.799-0.927)$ & $<0.001$ & 0.57 \\
\hline DeA_A $\left(H U^{*} \mathrm{~kg} / \mathrm{mg}\right)$ & $0.854(0.786-0.922)$ & $<0.001$ & 0.61 \\
\hline AbA_A (HU*kg/mg) & $0.826(0.758-0.894)$ & $<0.001$ & 0.58 \\
\hline FA_A (HU*kg/mg) & $0.747(0.654-0.839)$ & $<0.001$ & 0.51 \\
\hline IVC_A (HU*kg/mg) & $0.455(0.334-0.575)$ & 0.56 & 0.36 \\
\hline PA_V $\left(H U^{*} \mathrm{~kg} / \mathrm{mg}\right)$ & $0.773(0.663-0.858)$ & $<0.001$ & 0.30 \\
\hline AsA_V $\left(H U^{*} \mathrm{~kg} / \mathrm{mg}\right)$ & $0.763(0.671-0.856)$ & $<0.001$ & 0.37 \\
\hline DeA_V $\left(H U^{*} \mathrm{~kg} / \mathrm{mg}\right)$ & $0.750(0.663-0.836)$ & $<0.001$ & 0.34 \\
\hline AbA_V $\left(H U^{*} \mathrm{~kg} / \mathrm{mg}\right)$ & $0.756(0.665-0.847)$ & $<0.001$ & 0.32 \\
\hline FA_V (HU*kg/mg) & $0.749(0.652-0.847)$ & $<0.001$ & 0.32 \\
\hline IVC_V (HU*kg/mg) & $0.692(0.584-0.799)$ & $<0.001$ & 0.25 \\
\hline
\end{tabular}

AUROC, area under the receiver operating characteristic curve; CTAV, computed tomography attenuation values; $\mathrm{Cl}$, confidence interval; PA_A, pulmonary artery in the arterial phase; AsA_A, ascending aorta in the arterial phase; DeA_A, descending aorta in the arterial phase; AbA_A, abdominal aorta in the arterial phase; FA_A, femoral artery in the arterial phase; IVC_A, inferior vena cava in the arterial phase; PA_V, pulmonary artery in the portal venous phase; AsA_V, ascending aorta in the portal venous phase; DeA_V, descending aorta in the portal venous phase; AbA_V, abdominal aorta in the portal venous phase; FA_V, femoral artery in the portal venous phase; IVC_V, inferior vena cava in the portal venous phase.

a)The CTAV of major vessels was adjusted according to the iodine dose per body weight.

\section{DISCUSSION}

In this study, we found a strong association between initial enhanced CTAV of the aorta and the necessity for MT in major blunt trauma patients. A simple measurement of CTAV of the ascending 

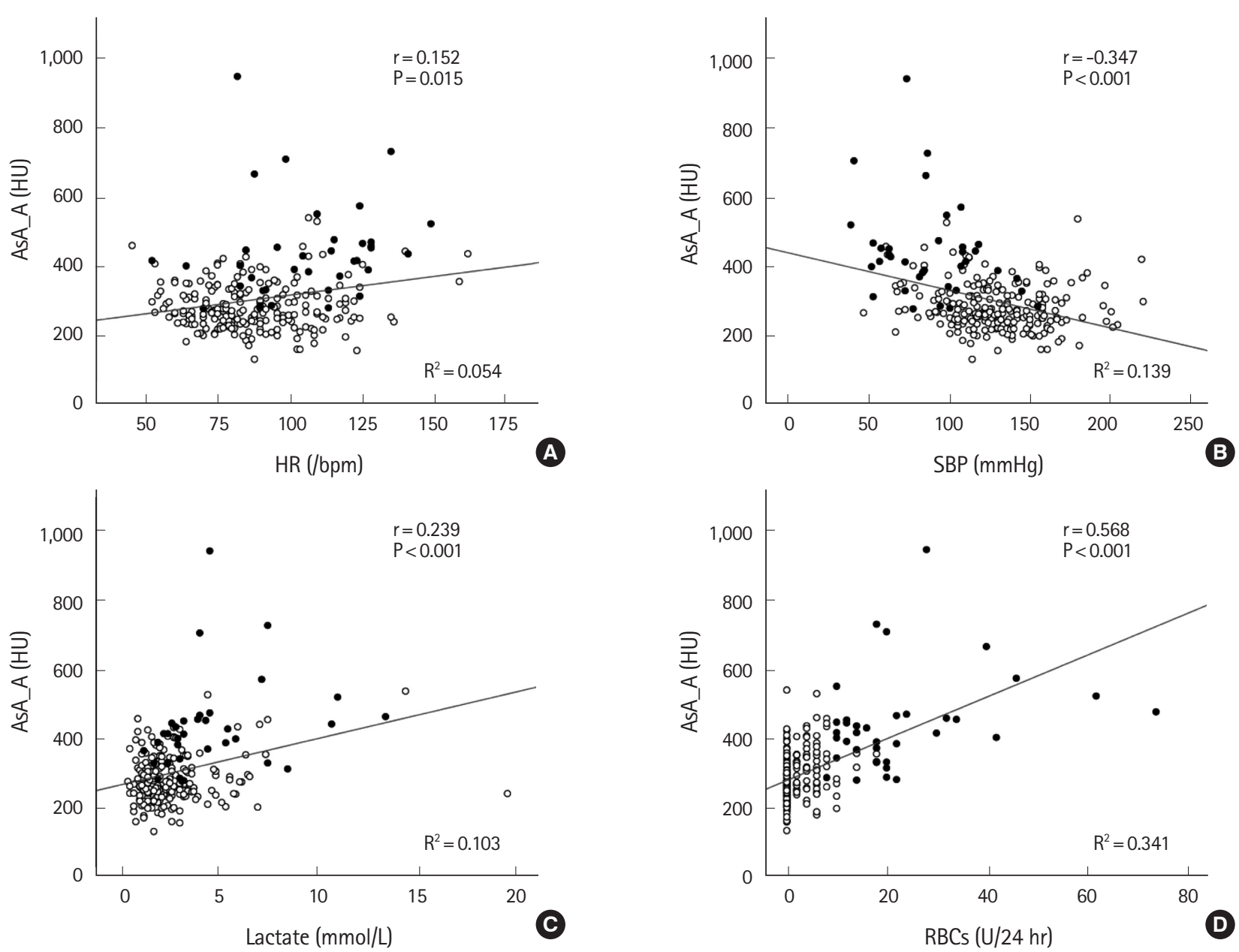

Fig. 2. Correlation between computed tomography attenuation values of the ascending aorta in the arterial phase and heart rate (HR) (A), systolic blood pressure SBP (B), lactate level on arrival (C), or requirement of red blood cells (RBCs) within 24 hours after arrival (D). Filled circles show the massive transfusion group $(n=36)$ and open circles show the no massive transfusion group $(n=218)$. The diagonal line represents a linear fit of the data. AsA_A, ascending aorta in the arterial phase.

or descending aorta in the arterial phase, which had the highest interobserver agreement and accuracy, could predict the requirement for MT. A higher CTAV of the aorta should alert the trauma surgeon or emergency physician to activate their MT protocol.

Currently, whole-body CT scanning, which includes a contrastenhanced scan of the torso, at initial assessment has been suggested to contribute to lower overall mortality than selective CT scanning in trauma patients. ${ }^{23} \mathrm{~A}$ recent study in Japan showed that CT examination was performed in more than $90 \%$ of unstable blunt trauma patients during initial management. ${ }^{24}$ The whole-body CT scan has become an initial critical diagnostic tool in major trauma patients. ${ }^{25}$ Examination at initial assessment is crucial as it can reveal unforeseen bleeding sources and help determine the appropriate hemostatic procedure according to priority. ${ }^{13}$ However, despite the widespread use of $\mathrm{CT}$, its findings have not been used to predict the need for MT, except for the presence of intra-abdominal free fluid collection, IVC diameter, or patterns of pelvic ring fracture and the presence of contrast extravasation in the region. ${ }^{9,15,26}$ Persistent appearance of a higher density of intravascular contrast material in trauma patients with severe hemorrhage caused us to investigate the association between CTAV and the need for MT.

Enhancement quality of the major vessels is mainly affected by cardiac output and body weight under conditions of a constant protocol. ${ }^{27} \mathrm{~A}$ previous report showed that cardiac output obtained via dynamic CT of the main pulmonary artery correlated well with cardiac output determined using the thermodilution method via right heart catheterization. ${ }^{27}$ Another report described a negative correlation between CTAV and cardiac output obtained via echocardiography. ${ }^{28}$ Our findings show that higher enhanced CTAV of the aorta in patients who require MT indicates lower cardiac output due to ongoing bleeding. No correlation between CTAV at the 

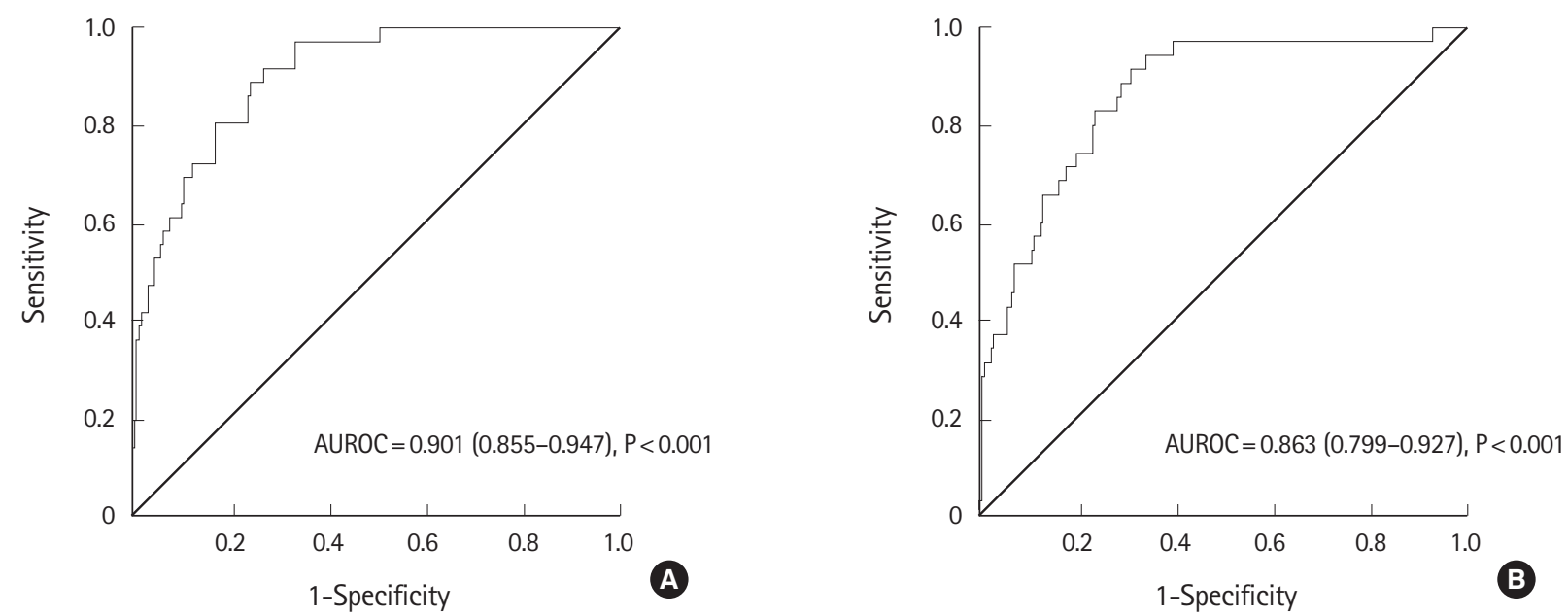

Fig. 3. Receiver operating characteristic curve of absolute computed tomography attenuation values at the descending aorta in the arterial phase for prediction of massive transfusion (A) and receiver operating characteristic curve of computed tomography attenuation values adjusted according to the iodine dose per body weight at the ascending aorta in the arterial phase for prediction of massive transfusion (B). AUROC, area under the receiver operating characteristic curve.

IVC in the arterial phase and MT was observed, which was considered as insufficient enhancement of the IVC. ${ }^{29}$

In patients with traumatic hypovolemic shock, enhancement patterns of solid abdominal organs have been investigated, and preserved enhancement of the adrenal gland, prolonged enhancement of the pancreas, or decreased enhancement of the renal medulla have been found. ${ }^{16,30}$ However, the detailed characteristics of the cohort or variable clinical parameters were unknown, and enhancement patterns varied depending on the severity of shock. ${ }^{30}$ Notably, we showed that the lower the SBP and the higher the lactate level on arrival, the more likely the increased enhanced CTAV of the aorta. However, an important limitation must be acknowledged, that is, the clinical parameters and enhanced CTAV of major vessels were not measured simultaneously.

We measured the CTAVs of six major vessel points to examine their predictive capability and reliability. Among them, increased absolute CTAV of the ascending or descending aorta in the arterial phase and its adjusted values according to iodine dose per body weight was revealed to be a highly accurate and reliable predictor of the need for MT. A relatively lower interobserver agreement of CTAV at the IVC may be explained by insufficient mixing of contrast material and vascular collapse due to hypovolemic shock. ${ }^{15,29}$

Our study had several limitations. First, the total iodine dose per body weight varied. The body weight and iodine dose administered are the most important factors affecting the magnitude of vascular contrast enhancement. ${ }^{19}$ Other affecting factors include the duration of injection and scan delay (i.e., the time interval from the start of contrast material injection to the initiation of scan), which were constant in all patients in the present study. ${ }^{19}$ In a clinical trauma care setting, the exact body weight is unavailable. Although the iodine dose was adjusted by estimating body weight, there was no significant difference with respect to total iodine dose per body weight between the MT group and no MT group. Also, the same results were obtained using both absolute CTAV and its values adjusted according to the iodine dose per body weight. Second, institutions use different CT scanners and different dynamic $\mathrm{CT}$ protocols, indicating difficulty of generalization, particularly in terms of absolute CTAV. Third, cardiac function, which affects the degree of contrast enhancement, particularly in a patient with heart failure, was not evaluated. Fourth, the time interval between patient arrival to CT scan was not accounted for, which would have been a relevant factor in our study. Finally, our study was a single-center retrospective design. Further large studies are warranted to validate our findings.

In conclusion, initial elevated enhanced CTAV of the aorta is a predictor of the need for MT. Given the high interobserver agreement and AUROC, a simple measurement of CTAV at the ascending or descending aorta in the arterial phase may help in determining the need for MT in patients with major blunt trauma.

\section{CONFLICT OF INTEREST}

No potential conflict of interest relevant to this article was reported.

\section{REFERENCES}

1. Dutton RP, Stansbury LG, Leone S, Kramer E, Hess JR, Scalea 
TM. Trauma mortality in mature trauma systems: are we doing better? An analysis of trauma mortality patterns, 19972008. J Trauma 2010;69:620-6.

2. Huber-Wagner $S$, Lefering $R$, Qvick LM, et al. Effect of wholebody CT during trauma resuscitation on survival: a retrospective, multicentre study. Lancet 2009;373:1455-61.

3. Hoyt DB, Bulger EM, Knudson MM, et al. Death in the operating room: an analysis of a multi-center experience. J Trauma 1994;37:426-32.

4. Holcomb JB, Jenkins $D$, Rhee $P$, et al. Damage control resuscitation: directly addressing the early coagulopathy of trauma. J Trauma 2007;62:307-10.

5. Hagiwara $A$, Kushimoto $S$, Kato $H$, et al. Can early aggressive administration of fresh frozen plasma improve outcomes in patients with severe blunt trauma? A report by the Japanese Association for the Surgery of Trauma. Shock 2016;45:495501.

6. Teixeira PG, Inaba K, Hadjizacharia P, et al Preventable or potentially preventable mortality at a mature trauma center. J Trauma 2007;63:1338-46.

7. Yucel $N$, Lefering $R$, Maegele M, et al. Trauma Associated Severe Hemorrhage (TASH)-Score: probability of mass transfusion as surrogate for life threatening hemorrhage after multiple trauma. J Trauma 2006;60:1228-36.

8. Nunez TC, Voskresensky IV, Dossett LA, Shinall R, Dutton WD, Cotton BA. Early prediction of massive transfusion in trauma: simple as ABC (assessment of blood consumption)? J Trauma 2009;66:346-52.

9. Rainer $T H, H o A M$, Yeung JH, et al. Early risk stratification of patients with major trauma requiring massive blood transfusion. Resuscitation 2011;82:724-9.

10. Vandromme MJ, Griffin RL, McGwin G Jr, Weinberg JA, Rue LW 3rd, Kerby JD. Prospective identification of patients at risk for massive transfusion: an imprecise endeavor. Am Surg 2011; 77:155-61.

11. Ogura T, Nakamura Y, Nakano M, et al. Predicting the need for massive transfusion in trauma patients: the Traumatic Bleeding Severity Score. J Trauma Acute Care Surg 2014;76:124350.

12. Yumoto $T$, lida $A$, Hirayama T, et al. Immediate screening method for predicting the necessity of massive transfusions in trauma patients: a retrospective single-center study. J Intensive Care 2014;2:54.

13. Wada D, Nakamori Y, Yamakawa $K$, et al. Impact on survival of whole-body computed tomography before emergency bleeding control in patients with severe blunt trauma. Crit Care 2013;17:R178.
14. Johnson JJ, Garwe T, Albrecht RM, et al. Initial inferior vena cava diameter on computed tomographic scan independently predicts mortality in severely injured trauma patients. J Trauma Acute Care Surg 2013;74:741-5.

15. Takada $H$, Hifumi $T$, Yoshioka $H$, et al. Initial inferior vena cava diameter predicts massive transfusion requirements in blunt trauma patients: A retrospective cohort study. Am J Emerg Med 2018;36:1155-59.

16. Higashi H, Tamada T, Kanki A, Yamamoto A, Ito K. Hypovolemic shock complex: does the pancreatic perfusion increase or decrease at contrast-enhanced dynamic CT? Clin Imaging 2014; 38:31-4.

17. Rifkin RD, Mernoff DB. Noninvasive evaluation of pericardial effusion composition by computed tomography. Am Heart J 2005;149:1120-7.

18. Cetin MS, Ozcan Cetin EH, Ozdemir M, et al. Effectiveness of computed tomography attenuation values in characterization of pericardial effusion. Anatol J Cardiol 2017;17:322-7.

19. Bae KT. Intravenous contrast medium administration and scan timing at CT: considerations and approaches. Radiology 2010; 256:32-61.

20. Mitra B, Cameron PA, Gruen RL, Mori A, Fitzgerald M, Street A. The definition of massive transfusion in trauma: a critical variable in examining evidence for resuscitation. Eur J Emerg Med 2011;18:137-42.

21. Savage SA, Zarzaur BL, Croce MA, Fabian TC. Redefining massive transfusion when every second counts. J Trauma Acute Care Surg 2013;74:396-400.

22. Savage SA, Sumislawski JJ, Bell TM, Zarzaur BL. Utilizing groupbased trajectory modeling to understand patterns of hemorrhage and resuscitation. Ann Surg 2016;264:1135-41.

23. Caputo ND, Stahmer C, Lim G, Shah K. Whole-body computed tomographic scanning leads to better survival as opposed to selective scanning in trauma patients: a systematic review and meta-analysis. J Trauma Acute Care Surg 2014;77:534-9.

24. Tsutsumi Y, Fukuma S, Tsuchiya A, et al. Computed tomography during initial management and mortality among hemodynamically unstable blunt trauma patients: a nationwide retrospective cohort study. Scand J Trauma Resusc Emerg Med 2017;25:74.

25. Huber-Wagner $S$, Kanz KG, Hanschen $M$, van Griensven $M$, Biberthaler $\mathrm{P}$, Lefering R. Whole-body computed tomography in severely injured patients. Curr Opin Crit Care 2018;24:5561.

26. Ohmori T, Matsumoto T, Kitamura $T$, et al. Scoring system to predict hemorrhage in pelvic ring fracture. Orthop Traumatol Surg Res 2016;102:1023-8. 
27. Tomizawa N, Komatsu S, Akahane M, Torigoe R, Kiryu S, Ohtomo K. Influence of hemodynamic parameters on coronary artery attenuation with 320-detector coronary CT angiography. Eur J Radiol 2012;81:230-3.

28. Pienn M, Kovacs G, Tscherner M, et al. Determination of cardiac output with dynamic contrast-enhanced computed tomography. Int J Cardiovasc Imaging 2013;29:1871-8.
29. Smillie RP, Shetty M, Boyer AC, Madrazo B, Jafri SZ. Imaging evaluation of the inferior vena cava. Radiographics 2015;35: 578-92.

30. Higashi $H$, Kanki $A$, Watanabe $S$, et al. Traumatic hypovolemic shock revisited: the spectrum of contrast-enhanced abdominal computed tomography findings and clinical implications for its management. Jpn J Radiol 2014;32:579-84. 